\title{
Stochastic Fractional Programming Approach to a Mean and Variance Model of a Transportation Problem
}

\author{
V. Charles, ${ }^{1}$ V. S. S. Yadavalli, ${ }^{2}$ M. C. L. Rao, ${ }^{3}$ and P. R. S. Reddy ${ }^{3}$ \\ ${ }^{1}$ CENTRUM Católica, Escuela de Graduados de Negocios, Pontificia Universidad Católica del Perú, \\ Lima 33, Peru \\ ${ }^{2}$ Department of Industrial and Systems Engineering, University of Pretoria, \\ Pretoria 0002, South Africa \\ ${ }^{3}$ Department of Statistics, Sri Venkateswara University, Tirupati, A.P. 517502, India
}

Correspondence should be addressed to V. S. S. Yadavalli, sarma.yadavalli@up.ac.za

Received 2 March 2010; Revised 3 November 2010; Accepted 23 February 2011

Academic Editor: J. J. Judice

Copyright (C) 2011 V. Charles et al. This is an open access article distributed under the Creative Commons Attribution License, which permits unrestricted use, distribution, and reproduction in any medium, provided the original work is properly cited.

In this paper, we propose a stochastic programming model, which considers a ratio of two nonlinear functions and probabilistic constraints. In the former, only expected model has been proposed without caring variability in the model. On the other hand, in the variance model, the variability played a vital role without concerning its counterpart, namely, the expected model. Further, the expected model optimizes the ratio of two linear cost functions where as variance model optimize the ratio of two non-linear functions, that is, the stochastic nature in the denominator and numerator and considering expectation and variability as well leads to a non-linear fractional program. In this paper, a transportation model with stochastic fractional programming (SFP) problem approach is proposed, which strikes the balance between previous models available in the literature.

\section{Introduction}

The transportation engineering problem is one of the most primitive applications of linear programming problems. The basic transportation problem was initially developed by Hitchcock [1] and has grown to the stage wherein supply chain management uses it significantly. Even one can say that supply chain's success is closely linked to the appropriate use of transportation. Linear fractional transportation problem was first discussed by Swarup [2] and since then it did not receive much attention. This paper deals with a fractional transportation model in which parameters involved in the model are probabilistic in nature. 
When the market demands for a commodity are stochastic in nature, the problem of scheduling shipments to a number of demand points from several supply points is a stochastic transportation problem [3]. Jörnsten et al. [4, 5] studied stochastic transportation model for petroleum transport and proposed a cross-decomposition algorithm to solve the said problem. The stochastic transportation problem can be formulated as a convex transportation problem with nonlinear objective function and linear constraints. Holmberg [6] compared different methods based on decomposition techniques and linearization techniques for this problem; Holmberg tried to find the most efficient method or combination of methods. Holmberg also discussed and tested a separable programming approach, the Frank-Wolfe method with and without modifications, the new technique of mean value cross-decomposition, and the more well-known Lagrangian relaxation with subgradient optimization, as well as combinations of these approaches.

Ratio optimization problems are commonly called fractional programs. One of the earliest fractional programs is an equilibrium model for an expanding economy introduced by Von Neumann in 1937, at a time when linear programming hardly existed. The linear and nonlinear models of fractional programming problems have been studied by Charnes and Cooper [7] and Dinkelbach [8]. The fractional programming problems have been studied extensively by many researchers. Mjelde [9] maximized the ratio of the return and the cost in resource allocation problems; Kydland [10], on the other hand, maximized the profit per unit time in a cargo-loading problem. Arora and Ahuja [11] discussed a fractional bulk transportation problem in which the numerator is quadratic in nature and the denominator is linear.

Stochastic fractional programming (SFP) offers a way to deal with planning in situations where the problem data is not known with certainty. Such situations arise where technological aspects of the system under study may be highly complicated or incapable of being observed completely. Stochastic Programming and Fractional Programming constitute two of the more vibrant areas of research in optimization. Both areas have blossomed into fields that have solid mathematical foundations, reliable algorithms and software, and a plethora of applications that continue to challenge current state-of-art computing resources. For various reasons, these areas have matured independently. Many of the existing procedures that are of practical importance for solving stochastic programming and fractional programming problems rely mostly on simplified assumptions. Wide range of applications of stochastic and fractional programming can be seen in [12-17].

A constrained linear stochastic fractional programming (LSFP) problem involves optimizing the ratio of two linear functions subject to some constraints in which at least one of the problem data is random in nature with nonnegative constraints on the variables. In addition, some of the constraints may be deterministic.

The LSFP framework attempts to model uncertainty in the data by assuming that the input or a part thereof is specified by a probability distribution, rather than being deterministic. Gupta [18] described a model on capacitated stochastic transportation problem, which maximizes profitability. LSFP has been extensively studied by Gupta et al. $[19,20]$ and Charles et al. [14-17, 21-31], the concepts of LSFP are available in [21, 22], various algorithms to solve LSFP have been discussed in $[23,26,28,29]$, financial derivative applications of nonlinear SFP are studied in $[25,27]$, and multiobjective LSFP problems are discussed in [24, 30]. Charles and Dutta [30] discusses an application to assembled printed circuit board of multi-objective LSFP, and an algorithm to identify redundant fractional objective function in multi-objective SFP is clearly discussed in [31, 32]. 
In this paper, a special class of transportation problems has been considered wherein the stochastic fractional programming (SFP) is the handy technique to optimize the transportation problem. The said special class of uncapacitated transportation problems has two distinct cost matrices in which costs involved in the problem are random in nature and are assumed to follow normal distribution, and the demand vector under study is also random wherein the demand vector is assumed to follow probability distributions like normal and uniform. The proposed meanvariance model attempts to optimize the profit over shipping cost under uncertain environment, subject to regular supply constraints along with stochastic demand constraints.

The organization of this paper is as follows. Section 2 discusses the uncapacitated transportation problem of SFP along with some basic assumptions. A deterministic equivalent of probabilistic demand constraints are described in Section 3 along with explanation for some of the preliminary properties of transportation problem of SFP and expectation, and also variance and mean-variance models for the uncapacitated transportation problem of SFP are established. In this Section 4 provides an algorithm to solve this problem. Discussion on this paper with a summary and recommendations for future research is in Section 5.

\section{The UnCapacitated Transportation Problem of LSFP}

This section deals with the uncapacitated TP of LSFP for the distribution of a single homogenous commodity from $m$ sources to $n$ destinations, where the demand for the commodity at each of the $n$ destinations is a random variable. An uncapacitated TP of LSFP in a criterion space is defined as follows:

$$
\operatorname{maximize} R(X)=\frac{N(X)+\alpha}{D(X)+\beta}=\frac{\sum_{i=1}^{m} \sum_{j=1}^{n} p_{i j} x_{i j}+\alpha}{\sum_{i=1}^{m} \sum_{j=1}^{n} c_{i j} x_{i j}+\beta^{\prime}}
$$

subject to

$$
\begin{aligned}
& \sum_{j=1}^{n} x_{i j} \leq a_{i}, \quad i=1,2, \ldots, m, \\
& \sum_{i=1}^{m} x_{i j}=r_{j}, \quad j=1,2, \ldots, n,
\end{aligned}
$$

where $0 \leq X_{m \times n}=\left\|x_{i j}\right\| \in R^{m \times n}$ is a feasible set, $S=\left\{X \mid(2.2)-(2.3), X \geq 0, X \in R^{m \times n}\right\}$ is nonempty, convex, and compact set in $R^{m \times n}, x_{i j}$ is an unknown quantity of the good shipped from supply point $i$ to demand point $j$, profit matrix $N_{m \times n}=\left\|p_{i j}\right\|$ which determines the profit $p_{i j} \sim N\left(u_{p_{i j}}, s_{p_{i j}}^{2}\right)$ gained from shipment from $i$ to $j$, cost matrix $D_{m \times n}=\left\|c_{i j}\right\|$ which determines the cost $c_{i j} \sim N\left(u_{c_{i j}}, s_{c_{i j}}^{2}\right)$ per unit of shipment from $i$ to $j$, the denominator function $D(X)+\beta$ is assumed to be positive throughout the constraint set, scalars $\alpha, \beta$, which determines some constant profit and cost, respectively, supply point $i$ must have $a_{i}$ units available, stochastic demand point $j$ must obtain $1-l_{j}$ level of $r_{j}$ units, and $1-l_{j}\left(0<l_{j}<1\right)$ is the least probability with which $j$ th stochastic demand constraint is satisfied. 
Stochastic equation (2.3) can be rewritten as follows:

$$
\begin{aligned}
& \operatorname{Pr}\left[\sum_{i=1}^{m} x_{i j} \geq r_{j}\right] \geq 1-l_{j}, \quad j=1,2, \ldots, n, \\
& \operatorname{Pr}\left[\sum_{i=1}^{m} x_{i j} \leq r_{j}\right] \geq 1-l_{j}, \quad j=1,2, \ldots, n .
\end{aligned}
$$

Assumption 2.1. (a) The values of every point of supply and demand are positive.

(b) Total supply is not less than total demand.

(c) Noninteger solutions are acceptable.

\section{Deterministic Equivalents of Probabilistic Demand Constraints and E-Model}

Let $r_{j}$ be a random variable in constraint (2.4) that follows $N\left(u_{r_{j}}, s_{r_{j}}^{2}\right), j=1,2, \ldots, n$, where $u_{r_{j}}$ is the $j$ th mean and $s_{r_{j}}^{2}$ is the $j$ th variance. The $j$ th deterministic demand constraint (2.4) is obtained from Charles and Dutta [21] and is given as follows:

$$
\operatorname{Pr}\left[\sum_{i=1}^{m} x_{i j} \geq r_{j}\right] \geq 1-l_{j} \quad \text { (or) } \operatorname{Pr}\left[r_{j} \leq \sum_{i=1}^{m} x_{i j}\right] \geq 1-l_{j} \quad \text { (or) } \quad \operatorname{Pr}\left(Z_{j} \leq z_{j}\right) \geq 1-l_{j}
$$

where $Z_{j}=\left(r_{j}-u_{r_{j}}\right) / s_{r_{j}}$ follows standard normal distribution and $z_{j}=\left(\sum_{i=1}^{m} x_{i j}-u_{r_{j}}\right) / s_{r_{j}}$. Thus, $\phi\left(z_{j}\right) \geq \phi\left(K_{1-l_{j}}\right)$, where $1-l_{j}=\phi\left(K_{1-l_{j}}\right)$, is the cumulative distribution function of standard normal distribution. Clearly, $\phi(\cdot)$ is a nondecreasing continuous function, hence $z_{j} \geq K_{1-l_{j}}$. The $j$ th deterministic demand constraint (2.4) is as follows:

$$
\sum_{i=1}^{m} x_{i j} \geq u_{r_{j}}+K_{1-l_{j}} s_{r_{j}}
$$

Similar to constraint (3.2), one can obtain the constraint given below from (2.5):

$$
\sum_{i=1}^{m} x_{i j} \leq u_{r_{j}}+K_{l_{j}} s_{r_{j}}
$$

Inequalities (3.2) and (3.3) can be combined as follows:

$$
u_{r_{j}}+K_{1-l_{j}} s_{r_{j}} \leq \sum_{i=1}^{m} x_{i j} \leq u_{r_{j}}+K_{l_{j}} s_{r_{j}}
$$

Let $r_{j}$ be the uniform random variable which ranges from $u_{j}^{\text {low }}$ to $u_{j}^{\text {up }}$, that is, $r_{j} \sim U\left(u_{j}^{\text {low }}, u_{j}^{\text {up }}\right)$, the probabilistic demand constraint in system (2.1) is equivalent to $\sum_{i=1}^{m} x_{i j} \geq \tau_{j}$, where 
$l_{j}^{\prime}=1-l_{j}$, and $\int_{\tau_{j}}^{u_{j}^{\text {up }}}\left(d x /\left(u_{j}^{\text {up }}-u_{j}^{\text {low }}\right)\right)=l_{j}^{\prime}$, that is, $\tau_{j}=l_{j} u_{j}^{\text {up }}+l_{j}^{\prime} u_{j}^{\text {low }}$. Hence, the deterministic equivalent of the $j$ th probabilistic demand constraint (2.4) is

$$
\sum_{i=1}^{m} x_{i j} \geq l_{j} u_{j}^{\text {up }}+l_{j}^{\prime} u_{j}^{\text {low }}
$$

Similar to (3.5) one can obtain the constraint given below from (2.5):

$$
\sum_{i=1}^{m} x_{i j} \leq l_{j} u_{j}^{\text {low }}+l_{j}^{\prime} u_{j}^{\text {up }}
$$

Constraints (3.5) and (3.6) can be combined as follows:

$$
l_{j} u_{j}^{\text {up }}+l_{j}^{\prime} u_{j}^{\text {low }} \leq \sum_{i=1}^{m} x_{i j} \leq l_{j} u_{j}^{\text {low }}+l_{j}^{\prime} u_{j}^{\text {up }}
$$

Definition 3.1. If the total supply lies in the interval of total deterministic demand, the transportation problem of SFP has feasible solutions.

Case 1. The normally distributed demand is $\sum_{j=1}^{n}\left(u_{r_{j}}+K_{1-l_{j}} s_{r_{j}}\right) \leq \sum_{i=1}^{m} a_{i} \leq \sum_{j=1}^{n}\left(u_{r_{j}}+K_{l_{j}} s_{r_{j}}\right)$.

Case 2. Uniformly distributed demand the $\sum_{j=1}^{n}\left(l_{j} u_{j}^{\text {up }}+l_{j}^{\prime} u_{j}^{\text {low }}\right) \leq \sum_{i=1}^{m} a_{i} \leq \sum_{j=1}^{n}\left(l_{j} u_{j}^{\text {low }}+l_{j}^{\prime} u_{j}^{\text {up }}\right)$.

Lemma 3.2. The transportation problem of SFP always has a feasible solution, that is, feasible set $S$ is nonempty.

Lemma 3.3. The set of feasible solutions is bounded.

Lemma 3.4. The transportation problem of SFP is solvable.

The proof of the above said properties when demand follows normal distribution are as follows: Let $x_{i j}^{*}$ be defined as

$$
\frac{a_{i}\left(u_{r_{j}}+K_{1-l_{j}} s_{r_{j}}\right)}{T_{1}} \leq x_{i j}^{*} \leq \frac{a_{i}\left(u_{r_{j}}+K_{l_{j}} s_{r_{j}}\right)}{T_{2}}, \quad i=1,2, \ldots, m, j=1,2, \ldots, n,
$$

where $T_{1}=\sum_{j=1}^{n}\left(u_{r_{j}}+K_{1-l_{j}} s_{r_{j}}\right), T_{2}=\sum_{j=1}^{n}\left(u_{r_{j}}+K_{l_{j}} s_{r_{j}}\right)$ are positive.

Substituting $x_{i j}^{*}$ for the supply and demand constraints, that is, from constraints (2.2) and (2.4), the following can be obtained:

$$
\begin{aligned}
& \sum_{j=1}^{n} x_{i j}^{*} \leq \sum_{j=1}^{n} \frac{a_{i}\left(u_{r_{j}}+K_{1-l_{j}} s_{r_{j}}\right)}{T_{1}}=\frac{a_{i}}{T_{1}} \sum_{j=1}^{n}\left(u_{r_{j}}+K_{1-l_{j}} s_{r_{j}}\right)=a_{i}, \quad i=1,2, \ldots, m, \\
& \sum_{j=1}^{n} x_{i j}^{*} \geq \sum_{j=1}^{n} \frac{a_{i}\left(u_{r_{j}}+K_{l_{j}} s_{r_{j}}\right)}{T_{2}}=\frac{a_{i}}{T_{2}} \sum_{j=1}^{n}\left(u_{r_{j}}+K_{l_{j}} s_{r_{j}}\right)=a_{i}, \quad i=1,2, \ldots, m,
\end{aligned}
$$


and hence $\sum_{j=1}^{n} x_{i j}^{*}=a_{i}$. From (3.8), one can obtain

$$
\begin{aligned}
& \sum_{i=1}^{m} \frac{a_{i}\left(u_{r_{j}}+K_{1-l_{j}} s_{r_{j}}\right)}{T_{1}} \leq \sum_{i=1}^{m} x_{i j}^{*} \leq \sum_{i=1}^{m} \frac{a_{i}\left(u_{r_{j}}+K_{l_{j}} s_{r_{j}}\right)}{T_{2}}, \\
& \frac{\left(u_{r_{j}}+K_{1-l_{j}} s_{r_{j}}\right)}{T_{1}} \sum_{i=1}^{m} a_{i} \leq \sum_{i=1}^{m} x_{i j}^{*} \leq \frac{\left(u_{r_{j}}+K_{l_{j}} s_{r_{j}}\right)}{T_{2}} \sum_{i=1}^{m} a_{i}, \\
& u_{r_{j}}+K_{1-l_{j}} s_{r_{j}} \leq \frac{\left(u_{r_{j}}+K_{1-l_{j}} s_{r_{j}}\right)}{T_{1}} \sum_{i=1}^{m} a_{i} \leq \sum_{i=1}^{m} x_{i j}^{*} \leq \frac{\left(u_{r_{j}}+K_{l_{j}} s_{r_{j}}\right)}{T_{2}} \sum_{i=1}^{m} a_{i} \leq u_{r_{j}}+K_{l_{j}} s_{r_{j}}, \\
& u_{r_{j}}+K_{1-l_{j}} s_{r_{j}} \leq \sum_{i=1}^{m} x_{i j}^{*} \leq u_{r_{j}}+K_{l_{j}} s_{r_{j}}, \quad j=1,2, \ldots, m .
\end{aligned}
$$

Hence, constraints (2.2) and (2.4) are satisfied by $x_{i j}^{*}$. Since from Assumption 2.1(a)(b) the constraint (3.2) it follows that $x_{i j}^{*}>0, i=1,2, \ldots, m, j=1,2, \ldots, n$, it becomes obvious that $x^{*}=\left(x_{i j}^{*}\right)$ is a feasible solution of the transportation problem of stochastic fractional programming. Thus it has been clearly shown that the feasible set $S$ is not empty.

Further, from (2.2), (3.4), and (3.7) along with nonnegativity constraints, it is clear that $0 \leq x_{i j}^{*} \leq a_{i}, i=1,2, \ldots, m ; j=1,2, \ldots, n$.

Expectation and variance of the profit and cost function of the probabilistic fractional objective function are defined as follows:

$$
\begin{aligned}
& E(N(X))=\sum_{i=1}^{m} \sum_{j=1}^{n} E\left(p_{i j}\right) x_{i j}+\alpha=\sum_{i=1}^{m} \sum_{j=1}^{n} u_{p i j} x_{i j}+\alpha, \\
& E(D(X))=\sum_{i=1}^{m} \sum_{j=1}^{n} E\left(c_{i j}\right) x_{i j}+\beta=\sum_{i=1}^{m} \sum_{j=1}^{n} u_{c i j} x_{i j}+\beta, \\
& V(N(X))=\sum_{i=1}^{m} \sum_{j=1}^{n} V\left(p_{i j}\right) x_{i j}=\sum_{i=1}^{m} \sum_{j=1}^{n} S_{p i j}^{2} x_{i j}^{2}, \\
& V(D(X))=\sum_{i=1}^{m} \sum_{j=1}^{n} V\left(c_{i j}\right) x_{i j}=\sum_{i=1}^{m} \sum_{j=1}^{n} S_{c i j}^{2} x_{i j}^{2} .
\end{aligned}
$$

Hence the deterministic fractional objective function is as follows:

$$
R^{E V}(X)=\frac{w_{1}\left(\sum_{i=1}^{m} \sum_{j=1}^{n} u_{p i j} x_{i j}+\alpha\right)+w_{2} \sqrt{\sum_{i=1}^{m} \sum_{j=1}^{n} S_{p i j}^{2} x_{i j}^{2}}}{w_{1}\left(\sum_{i=1}^{m} \sum_{j=1}^{n} u_{c i j} x_{i j}+\beta\right)+w_{2} \sqrt{\sum_{i=1}^{m} \sum_{j=1}^{n} S_{c i j}^{2} x_{i j}^{2}}}
$$

where $w_{1}$ and $w_{2}$ are preselected nonnegative numbers indicating the relative importance for optimization of the mean and the square root of the variance covariance matrix. The special 
cases corresponding to $w_{2}=0$ and $w_{1}=0$ are, respectively, known as the $E$-model and the $V$-model. The objective function (3.12) is very a well-known mean-variance model.

Since the numerator and denominator functions of the fractional objective function (3.12) are in Kataoka's [32] form and the denominator is assumed to be positive over the bounded feasible set $S$, it means that fractional objective function $R^{E V}(X)$ is also bounded over the same feasible set $S$, and hence it can be concluded that transportation problem of SFP is solvable.

The E-model for the uncapacitated TP of LSFP when demand follows normal distribution is as follows:

$$
\begin{array}{ll}
\text { maximize } & R^{E}(X)=\frac{\sum_{i=1}^{m} \sum_{j=1}^{n} u_{p i j} x_{i j}+\alpha}{\sum_{i=1}^{m} \sum_{j=1}^{n} u_{c i j} x_{i j}+\beta}, \\
\text { subject to } & \sum_{j=1}^{n} x_{i j} \leq a_{i}, \quad i=1,2, \ldots, m, \\
& u_{r_{j}}+K_{1-l_{j}} s_{r_{j}} \leq \sum_{i=1}^{m} x_{i j} \leq u_{r_{j}}+K_{l_{j}} s_{r_{j}}, \quad j=1,2, \ldots, n,
\end{array}
$$

where $0 \leq X_{m \times n}=\left\|x_{i j}\right\| \in R^{m \times n}$ is a feasible set, $S=\left\{X \mid(2.2)\right.$ and (3.4), $\left.X \geq 0, X \in R^{m \times n}\right\}$ is nonempty, convex and compact set in $R^{m \times n}, x_{i j}$ is an unknown quantity of the good shipped from supply point $i$ to demand point $j, R^{E}(X)$ is the fractional objective function defined as ratio of the profit function over the cost function, the profit and cost function is assumed to be positive throughout the constraint set, supply point $i$ must have at most $a_{i}$ units, deterministic demand point $j$ must obtain at least $u_{r_{j}}+K_{1-l_{j}} s_{r_{j}}$ units and at most $u_{r_{j}}+K_{l_{j}} s_{r_{j}}$ units. Similarly one can define $E$-model of system (2.1), when demand follows uniform distribution or/and normal distribution.

Lemma 3.5. This lemma is proposed with the $R^{E}(\cdot)$ being defined in the earlier section as the fractional objective function:

(1.1) $R^{E}(\lambda)$ is a convex function for $\lambda \in R$.

(1.2) $R^{E}(\lambda)$ is strictly decreasing function on $R$.

(1.3) $R^{E}(\lambda)$ is continuous function on $R$.

(1.4) The equation $R^{E}(\lambda)=0$ has unique solution, say $\lambda^{*}$.

(1.5) $R^{E}(\lambda) \geq 0$ for all $x \in S$.

Theorem 3.6. A necessary and sufficient condition for

$$
\lambda^{*}=\frac{\sum_{i=1}^{m} \sum_{j=1}^{n} u_{p i j} x_{i j}^{*}+\alpha}{\sum_{i=1}^{m} \sum_{j=1}^{n} u_{c i j} x_{i j}^{*}+\beta}=\underset{x \in S}{\operatorname{maximize}} \frac{\sum_{i=1}^{m} \sum_{j=1}^{n} u_{p i j} x_{i j}+\alpha}{\sum_{i=1}^{m} \sum_{j=1}^{n} u_{c i j} x_{i j}+\beta}
$$

is

$$
R^{E}\left(\lambda^{*}\right)=R^{E}\left(x^{*}, \lambda^{*}\right)=\underset{x \in S}{\operatorname{maximize}}\left[\sum_{i=1}^{m} \sum_{j=1}^{n} u_{p i j} x_{i j}+\alpha-\lambda^{*}\left(\sum_{i=1}^{m} \sum_{j=1}^{n} u_{c i j} x_{i j}+\beta\right)\right]=0 .
$$


Note. It may be noted that optimal solution $x^{*}$ may not be unique for the extremes (i.e., $\max / \min$ ). The $V$-model for the uncapacitated TP of SFP when demand follows normal distribution is as follows:

$$
\operatorname{maximize} \quad R^{\mathrm{V}}(\mathrm{X})=\frac{\sqrt{\sum_{\mathrm{i}=1}^{\mathrm{m}} \sum_{\mathrm{j}=1}^{\mathrm{n}} \mathrm{S}_{\mathrm{pij}}^{2} \mathrm{x}_{\mathrm{ij}}^{2}}}{\sqrt{\sum_{\mathrm{i}=1}^{\mathrm{m}} \sum_{\mathrm{j}=1}^{\mathrm{n}} \mathrm{S}_{\mathrm{cij}}^{2} \mathrm{x}_{\mathrm{ij}}^{2}}}
$$

subject to $\sum_{j=1}^{n} x_{i j} \leq a_{i}, i=1,2, \ldots, m, u_{r_{j}}+K_{1-l_{j}} s_{r_{j}} \leq \sum_{i=1}^{m} x_{i j} \leq u_{r_{j}}+K_{l_{j}} s_{r_{j}} j=1,2, \ldots, n$, where $0 \leq X_{m \times n}=\left\|x_{i j}\right\| \in R^{m \times n}$ is a feasible set, $S=\{X \mid(2.2)$ and (3.4), X $\geq 0, X \in$ $\left.R^{m \times n}\right\}$ is nonempty, convex, and compact set in $R^{m \times n}, x_{i j}$ is an unknown quantity of the good shipped from supply point $i$ to demand point $j, R^{V}(X)$ is the fractional objective function defined as ratio of standard deviation of the profit function over standard deviation of the cost function, the profit and cost function is assumed to be positive throughout the constraint set, supply point $i$ must have at most $a_{i}$ units, deterministic demand point $j$ must obtain at least $u_{r_{j}}+K_{1-l_{j}} s_{r_{j}}$ units and at most $u_{r_{j}}+K_{l_{j}} s_{r_{j}}$ units. Similarly one can define $V$-model of system (2.1) when demand follows uniform distribution or/and normal distribution.

Lemma 3.7. The following results are true.

(2.1) $R^{V 2}(\lambda)$ is a convex function for $\lambda \in R$.

(2.2) $R^{V 2}(\lambda)$ is strictly decreasing function on $R$.

(2.3) $R^{V 2}(\lambda)$ is continuous function on $R$.

(2.4) The equation $R^{V 2}(\lambda)=0$ has unique solution, say $\lambda^{*}$.

(2.5) $R^{V 2}(\lambda) \geq 0$ for all $x \in S$.

Theorem 3.8. A necessary and sufficient condition for

$$
\lambda^{*}=\frac{\sum_{i=1}^{m} \sum_{j=1}^{n} S_{p i j}^{2} x_{i j}^{2 *}}{\sum_{i=1}^{m} \sum_{j=1}^{n} S_{c i j}^{2} x_{i j}^{2 *}}=\underset{x \in S}{\operatorname{maximize}} \frac{\sum_{i=1}^{m} \sum_{j=1}^{n} S_{p i j}^{2} x_{i j}^{2}}{\sum_{i=1}^{m} \sum_{j=1}^{n} S_{c i j}^{2} x_{i j}^{2}}
$$

is

$$
R^{V 2}\left(\lambda^{*}\right)=R^{V 2}\left(x^{*}, \lambda^{*}\right)=\underset{x \in S}{\operatorname{optimize}}\left[\sum_{i=1}^{m} \sum_{j=1}^{n} S_{p i j}^{2} x_{i j}^{2}-\lambda^{*} \sum_{i=1}^{m} \sum_{j=1}^{n} S_{c i j}^{2} x_{i j}^{2}\right]=0 .
$$

Note. It may be noted that optimal solution $x^{*}$ may not be unique for the extremes (i.e., $\max / \mathrm{min}$ ). The mean-variance model for the uncapacitated TP of SFP when demand follows normal distribution is as follows:

$$
\operatorname{maximize} \quad \mathrm{R}^{\mathrm{EV}}(\mathrm{X})=\frac{\mathrm{w}_{1}\left(\sum_{\mathrm{i}=1}^{\mathrm{m}} \sum_{\mathrm{j}=1}^{\mathrm{n}} \mathrm{u}_{\mathrm{pij}} \mathrm{x}_{\mathrm{ij}}+\mathrm{ff}\right)+\mathrm{w}_{2} \sqrt{\sum_{\mathrm{i}=1}^{\mathrm{m}} \sum_{\mathrm{j}=1}^{\mathrm{n}} \mathrm{S}_{\mathrm{pij}}^{2} \mathrm{x}_{\mathrm{ij}}^{2}}}{\mathrm{w}_{1}\left(\sum_{\mathrm{i}=1}^{\mathrm{m}} \sum_{\mathrm{j}=1}^{\mathrm{n}} \mathrm{u}_{\mathrm{cij}} \mathrm{x}_{\mathrm{ij}}+\mathrm{fi}\right)+\mathrm{w}_{2} \sqrt{\sum_{\mathrm{i}=1}^{\mathrm{m}} \sum_{\mathrm{j}=1}^{\mathrm{n}} \mathrm{S}_{\mathrm{cij}}^{2} \mathrm{x}_{\mathrm{ij}}^{2}}}
$$


subject to $\sum_{j=1}^{n} x_{i j} \leq a_{i}, i=1,2, \ldots, m, u_{r_{j}}+K_{1-l_{j}} s_{r_{j}} \leq \sum_{j=1}^{m} x_{i j} \leq u_{r_{j}}+K_{l_{j}} s_{r_{j}}, j=1,2, \ldots, n$, where $0 \leq X_{m \times n}=\left\|x_{i j}\right\| \in R^{m \times n}$ is a feasible set, $S=\{X \mid(2.2)$ and (3.4), $X \geq 0, X \in$ $\left.R^{m \times n}\right\}$ is nonempty, convex, and compact set in $R^{m \times n}$, and $x_{i j}$ is an unknown quantity of the good shipped from supply point $i$ to demand point $j$. Similarly one can define meanvariance model of system (2.1) when demand follows uniform distribution or/and normal distribution.

Theorem 3.9. A necessary and sufficient condition for

$$
\begin{aligned}
\lambda^{*} & =\frac{w_{1}\left(\sum_{i=1}^{m} \sum_{j=1}^{n} u_{p i j} x_{i j}^{*}+\alpha\right)+w_{2} \sqrt{\sum_{i=1}^{m} \sum_{j=1}^{n} S_{p i j}^{2} x_{i j}^{2 *}}}{w_{1}\left(\sum_{i=1}^{m} \sum_{j=1}^{n} u_{c i j} x_{i j}^{*}+\beta\right)+w_{2} \sqrt{\sum_{i=1}^{m} \sum_{j=1}^{n} S_{c i j}^{2} x_{i j}^{2 *}}} \\
& =\underset{x \in S}{\operatorname{maximize}} \frac{w_{1}\left(\sum_{i=1}^{m} \sum_{j=1}^{n} u_{p i j} x_{i j}+\alpha\right)+w_{2} \sqrt{\sum_{i=1}^{m} \sum_{j=1}^{n} S_{p i j}^{2} x_{i j}^{2}}}{w_{1}\left(\sum_{i=1}^{m} \sum_{j=1}^{n} u_{c i j} x_{i j}+\beta\right)+w_{2} \sqrt{\sum_{i=1}^{m} \sum_{j=1}^{n} S_{c i j}^{2} x_{i j}^{2}}}
\end{aligned}
$$

is

$$
\begin{aligned}
R^{E V}\left(\lambda^{*}\right)= & R^{E V}\left(x^{*}, \lambda^{*}\right) \\
= & \underset{x \in S}{\operatorname{maximize}}\left[w_{1}\left(\sum_{i=1}^{m} \sum_{j=1}^{n} u_{p i j} x_{i j}+\alpha\right)+w_{2} \sqrt{\sum_{i=1}^{m} \sum_{j=1}^{n} S_{p i j}^{2} x_{i j}^{2}}\right. \\
& \left.-\lambda^{*}\left(w_{1}\left(\sum_{i=1}^{m} \sum_{j=1}^{n} u_{c i j} x_{i j}+\beta\right)+w_{2} \sqrt{\sum_{i=1}^{m} \sum_{j=1}^{n} S_{c i j}^{2} x_{i j}^{2}}\right)\right]=0 .
\end{aligned}
$$

\section{Algorithm: Sequential Linear Programming for TP of SFP}

(1) Start with an initial point $X^{(0)}$ and set the iteration number $t=0$ (there are many ways to get the initial guess $X^{(0)}$, one among is to solve $\left.\operatorname{maximize}_{x \in S} \sum_{i=1}^{m} \sum_{j=1}^{n} u_{p i j} x_{i j}\right)$.

(2) Decide the importance of mean and variance by means of assigning values to $w_{1}$ and $w_{2}$.

(3) Obtain

$$
\lambda^{(0)}=\frac{w_{1}\left(\sum_{i=1}^{m} \sum_{j=1}^{n} u_{p i j} x_{i j}+\alpha\right)+w_{2} \sqrt{\sum_{i=1}^{m} \sum_{j=1}^{n} S_{p i j}^{2} x_{i j}^{2}}}{w_{1}\left(\sum_{i=1}^{m} \sum_{j=1}^{n} u_{c i j} x_{i j}+\beta\right)+w_{2} \sqrt{\sum_{i=1}^{m} \sum_{j=1}^{n} S_{c i j}^{2} x_{i j}^{2}}} .
$$

(4) Linearize the constraint form of objective function about the points $\left(X^{(t)}, \lambda^{(t)}\right)$ as $R^{E V}(X, \lambda) \approx R^{E V}\left(X^{(t)}, \lambda^{(t)}\right)+\nabla R^{E V}\left(X^{(t)}, \lambda^{(t)}\right)^{T}\left(X-X^{(t)}, \lambda-\lambda^{(t)}\right)^{T}$. 
(5) Formulate the approximate TP of LSFP as

$\underset{x \in S}{\operatorname{maximize}} \lambda$ subject to $R^{E V}\left(X^{(t)}, \lambda^{(t)}\right)+\nabla R^{E V}\left(X^{(t)}, \lambda^{(t)}\right)^{T}\left(X-X^{(t)}, \lambda-\lambda^{(t)}\right)^{T}=0$.

(6) Solve the approximating TP of SFP to obtain the solution vector $X^{(t+1)}$ and scalar $\lambda^{(t+1)}$.

(7) Find $R^{E V}\left(X^{(t+1)}, \lambda^{(t+1)}\right)$.

(8) If $\left|R^{E V}\left(X^{(t+1)}, \lambda^{(t+1)}\right)\right| \leq \varepsilon$, where $\varepsilon$ is a prescribed small positive tolerance, all the demand and supply constraints can be assumed to have been satisfied. Hence stop the procedure by considering optimal $X$ is approximately equal to $X^{(t+1)}$, that is, $X^{\text {opt }}=X^{(t+1)}$.

(9) Else, once again linearize the constraint form of objective function about the points $\left(X^{(t+1)}, \lambda^{(t+1)}\right)$ as $R^{E V}(X, \lambda) \approx R^{E V}\left(X^{(t+1)}, \lambda^{(t+1)}\right)+\nabla R^{E V}\left(X^{(t+1)}, \lambda^{(t+1)}\right)^{T}$ $\left(X-X^{(t+1)}, \lambda-\lambda^{(t+1)}\right)^{T}$ and add this as an additional constraint to TP of SFP defined in step (4).

(10) Increment the iteration number by 1 and go to step (4).

\section{Discussion and Future Research}

A transportation model with stochastic programming approach is considered, and an algorithm to this effect has been presented. The reason to use SFP was to deal with planning in situations where the problem data is known only in the stochastic environment. Such situations arise in high technological complex systems. This proposed model would provide useful solution under those circumstances when the company likes to optimize the ratio of profit over the cost per unit of shipment in a way to meet the stochastic demands with a clear account for variation. This paper can be extended to an integer solution using branch and bound technique. Mixed model for TP of SFP and stochastic fractional recourse programming may be the interest of future research.

\section{Acknowledgments}

The authors are grateful to the Editors, anonymous referees for their valuable comments and suggestions. The author V. Charles is thankful to Carmen Mazzerini at the CENTRUM investigocion for her assistance. Thanks are due to NRF (South Africa) for their financial assistance to the author V. S. S. Yadavalli.

\section{References}

[1] F. L. Hitchcock, "The distribution of a product from several sources to numerous localities," Journal of Mathematics and Physics, vol. 20, pp. 224-230, 1941.

[2] K. Swarup, "Transportation technique in linear fractional functionals programming," Journal of The Royal Naval Scientific Service, vol. 21, no. 5, pp. 90-94, 1966. 
[3] A. C. Williams, "A stochastic transportation problem," Operations Research, vol. 11, pp. 759-770, 1963.

[4] K. Holmberg and K. O. Jörnsten, "Cross decomposition applied to the stochastic transportation problem," European Journal of Operational Research, vol. 17, no. 3, pp. 361-368, 1984.

[5] K. Jörnsten, R. Aboudi, Å Hallefjord et al., "A mathematical programming model for the development of petroleum fields and transport systems," European Journal of Operational Research, vol. 43, no. 1, pp. 13-25, 1989.

[6] K. Holmberg, "Efficient decomposition and linearization methods for the stochastic transportation problem," Computational Optimization and Applications, vol. 4, no. 4, pp. 293-316, 1995.

[7] A. Charnes and W. W. Cooper, "Programming with linear fractional functionals," Naval Research Logistics Quarterly, vol. 9, pp. 181-186, 1962.

[8] W. Dinkelbach, "On nonlinear fractional programming," Management Science, vol. 13, pp. 492-498, 1967.

[9] K. M. Mjelde, "Allocation of resources according to a fractional objective," European Journal of Operational Research, vol. 2, no. 2, pp. 116-124, 1978.

[10] F. Kydland, Simulation of Linear Operations, Institute of Shipping Research Norwegian School of Economics and Business Administration, Bergen, Norway, 1969.

[11] S. R. Arora and A. Ahuja, "Non-convex bulk transportation problem," International Journal of Management Science, vol. 7, no. 2, pp. 59-71, 2001.

[12] M. Jeeva, R. Rajagopal, and V. Charles, "Stochastic programming in manpower planning-cluster based optimum allocation of recruitments," in Advances in Stochastic Modelling, J. R. Artalejo and A. Krishnamoorthy, Eds., pp. 147-155, Notable Publications, New Jersey, NJ, USA, 2002.

[13] M. Jeeva, R. Rajagopal, V. Charles, and V. S. S. Yadavalli, "An application of stochastic programming with Weibull distribution-cluster based optimum allocation of recruitment in manpower planning," Stochastic Analysis and Applications, vol. 22, no. 3, pp. 801-812, 2004.

[14] V. Charles, Application of stochastic programming models to finance, Management of Cost, Finance and Human Resource for Good Governance, Karnataka Law Society's, IMER, pp. 106-115, 2005.

[15] V. Charles, A financial model for ELSS mutual fund schemes in India, management of cost, Finance and Human Resource for Good Governance, Karnataka Law Society's, IMER, pp. 127-136, 2005.

[16] V. Charles, "A stochastic goal programming model for capital rationing - carry forward of cash problem with mixed constraints," The ICFAI Journal of Applied Finance, vol. 11, no. 11\&12, pp. 37-48, 2005.

[17] V. Charles, "Reliability stochastic optimization-an application of stochastic integer programming$n$ stage series system with $m$ chance constraints," in Proceedings of the International Conference on Operations Research for Development, J. Shah, Ed., vol. 2, pp. 267-271, International Federation of Operational Research Society, 2005.

[18] S. N. Gupta, "A capacitated stochastic transportation problem for maximizing profitability," Ricerca Operativa, vol. 19, pp. 3-12, 1981.

[19] S. N. Gupta and K. Swarup, "Stochastic fractional functionals programming, Ricerca Operativa," Franco Angeli-Anno IX-Nuova Serien, vol. 10, pp. 65-78, 1979.

[20] S. N. Gupta, K. Swarup, and B. Lal, "Stochastic fractional programming with random technology matrix," Gujarat Statistical Review, vol. 3, pp. 23-34, 1981.

[21] V. Charles and D. Dutta, "Linear stochastic fractional programming with branch-and-bound technique," in Proceedings of the National Conference on Mathematical and Computational Methods, R. Nadarajan and P. R. Kandasamy, Eds., pp. 131-139, 2001.

[22] V. Charles, D. Dutta, and K. Appala Raju, "Linear stochastic fractional programming problem," in Proceedings of the International Conference on Mathematical Modelling, B. Singh, U. S. Gupta, G. S. Srivastava, T. R. Gulati, and V. K. Katiyar, Eds., pp. 211-217, Tata McGraw-Hill, 2001.

[23] V. Charles and D. Dutta, "Two level linear stochastic fractional programming problem with discrepancy vector," Journal of Indian Society of Statistics and Operations Research, vol. 23, no. 1-4, pp. 59-67, 2002.

[24] V. Charles and D. Dutta, "Bi-weighted multi-objective stochastic fractional programming problem with mixed constraints," in Proceedings of the 2nd National Conference on Mathematical and Computational Methods, pp. 29-36, Allied, New Delhi, India, 2003.

[25] V. Charles and D. Dutta, "Non-linear stochastic fractional programming models of financial derivatives- II," in Proceedings of the of International Conference on Business and Finance, N. Swain, D. K. Malhotra, and B. Roy, Eds., vol. 3, pp. 253-263, 2004.

[26] V. Charles and D. Dutta, "A method for solving linear stochastic fractional programming problem with mixed constraints," Acta Ciencia Indica, vol. 30, no. 3, pp. 497-506, 2004. 
[27] V. Charles and D. Dutta, "Non-linear stochastic fractional programming models of financial derivatives," The ICFAI Journal of Applied Finance, vol. 11, no. 6, pp. 5-13, 2005.

[28] V. Charles and D. Dutta, "Optimization of linear stochastic fractional programming problem using sign technique," Mathematical \& Computational Models, no. 28, pp. 302-341, 2005.

[29] V. Charles and D. Dutta, "A parametric approach to linear probabilistic fractional programming problems," Mathematical E Computational Models, no. 13, pp. 171-183, 2005.

[30] V. Charles and D. Dutta, "Extremization of multi-objective stochastic fractional programming problem," Annals of Operations Research, vol. 143, pp. 297-304, 2006.

[31] V. Charles and D. Dutta, "Identification of redundant objective functions in multi-objective stochastic fractional programming problems," Asia-Pacific Journal of Operational Research, vol. 23, no. 2, pp. 155170, 2006.

[32] S. Kataoka, “A stochastic programming model," Econometrica, vol. 31, pp. 181-196, 1963. 


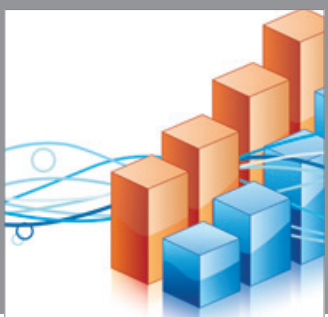

Advances in

Operations Research

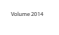

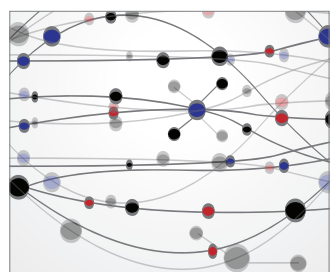

\section{The Scientific} World Journal
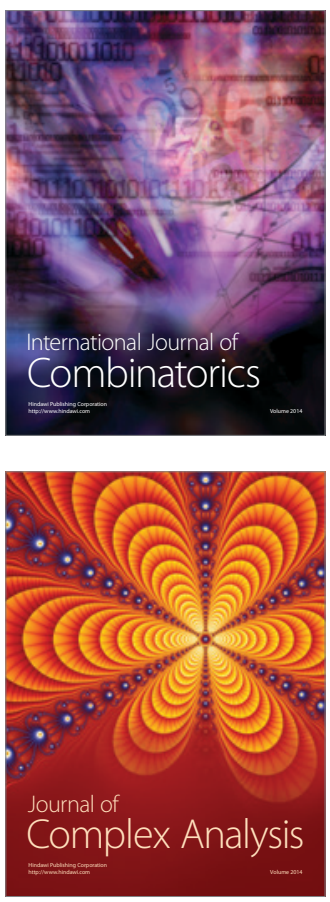

International Journal of

Mathematics and

Mathematical

Sciences
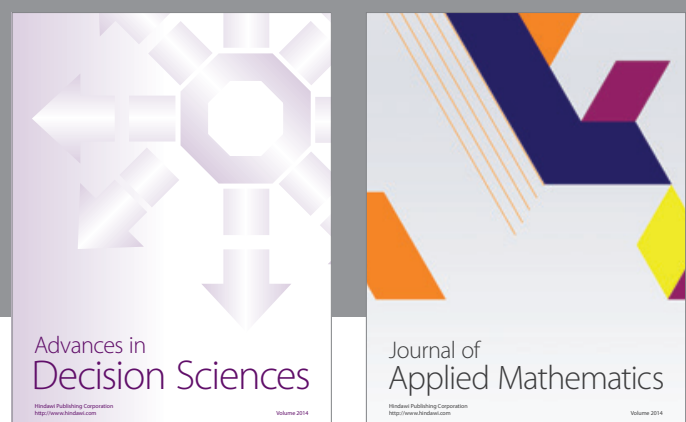

Journal of

Applied Mathematics
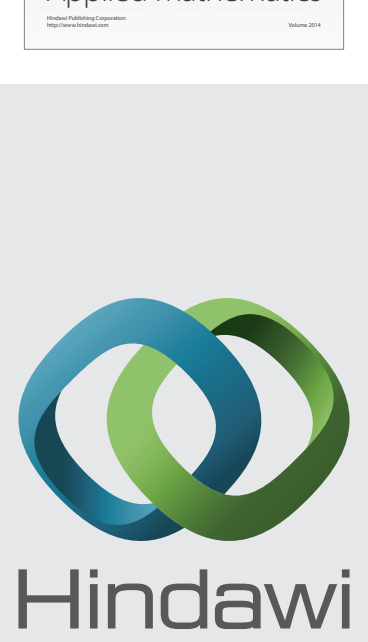

Submit your manuscripts at http://www.hindawi.com
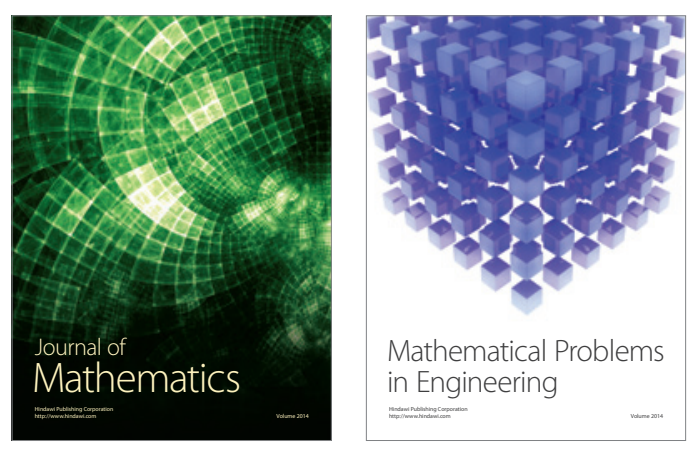

Mathematical Problems in Engineering
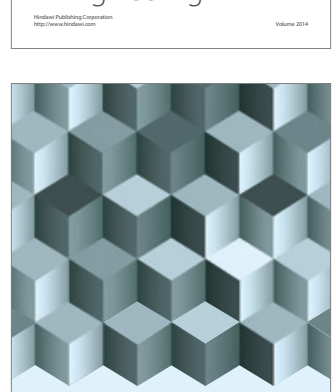

Journal of

Function Spaces
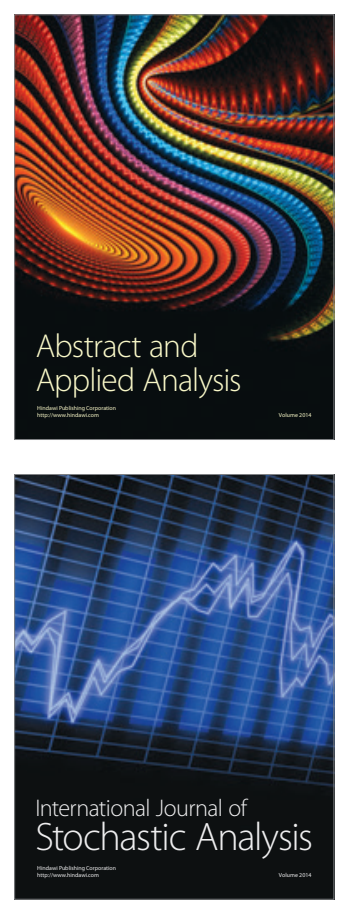

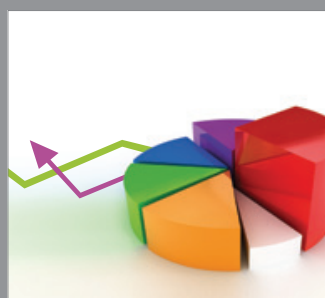

ournal of

Probability and Statistics

Promensencen
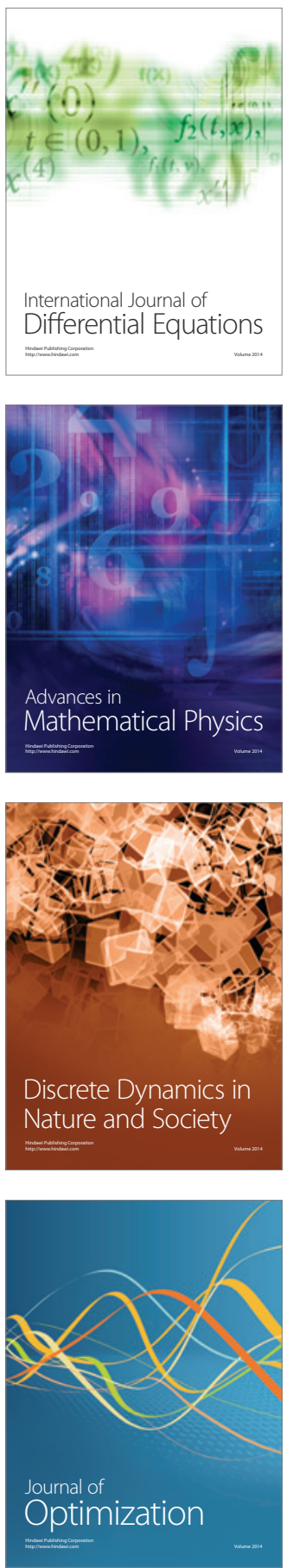